PROCEEDINGS OF THE

AMERICAN MATHEMATICAL SOCIETY

Volume 127, Number 11, Pages 3221-3227

S 0002-9939(99)04894-7

Article electronically published on April 27, 1999

\title{
ARENS REGULARITY OF WEAKLY SEQUENTIALLY COMPLETE BANACH ALGEBRAS
}

\author{
A. ÜLGER \\ (Communicated by Christopher D. Sogge)
}

\begin{abstract}
In this paper we prove the following result: Let $A$ be a nonunital Banach algebra with a bounded approximate identity. Then $A$ cannot be both Arens regular and weakly sequentially complete. The paper also contains some applications of this result.
\end{abstract}

\section{INTRODUCTION}

In this paper we prove a general result about the Arens regularity of weakly sequentially complete Banach algebras. As is well known, many of the Banach algebras that arise in harmonic analysis and other parts of mathematics are weakly sequentially complete (=WSC). Just to list some of them, let us mention the following ones:

a) Group algebras $L^{1}(G)$ and their weighted analogs $L^{1}(G ; \omega)$.

b) Semigroup algebras $\ell^{1}(S)$ and their weighted analogs $\ell^{1}(S ; \omega)$.

c) Hypergroup algebras $L(X)([\mathrm{D}]$ and $[\mathrm{J}])$.

d) The Fourier algebra $A(G)([\mathrm{E}])$ and, more generally, algebras that are predual of the von Neuman algebras.

e) Various kinds of measure algebras.

f) Reflexive algebras. $\ell^{1}$.

g) Certain sequence spaces with coordinatewise multiplication such as the algebra

h) The algebras obtained through certain Banach algebra constructions applied to the preceding ones.

Among these algebras, some are Arens regular but most are not. If one looks at the Arens regular ones, we see that they are either unital (e.g., $\ell^{1}(Z ; \omega)$, with $\omega(n)$ $=1+|n|[\mathrm{C}-\mathrm{Y}])$, or they do not have a bounded approximate identity (= b.a.i.) (e.g., $\left.\ell^{1}\right)$. This observation led us to wonder whether there exist nonunital WSC Banach algebras with a b.a.i. [U1, p. 395, Question 3]. The main result of this paper states that such an algebra does not exist. In other words, a nonunital Banach algebra with a b.a.i. cannot be both Arens regular and WSC. The group algebra $L^{1}(G)$ is WSC and has a b.a.i. but is Arens regular only if the group $G$ is finite. On the other hand, there exist many non-WSC and nonunital Arens regular

Received by the editors July 29, 1997 and, in revised form, January 22, 1998.

1991 Mathematics Subject Classification. Primary 46G10, 46H20.

Key words and phrases. Arens regularity, homomorphism.

This work was supported by a fund of the Turkish Academy of Sciences.

(c)1999 American Mathematical Society 
Banach algebras with a b.a.i. (e.g., $\left.C_{o}(R)\right)$ as well as WSC ones without b.a.i. (e.g., reflexive algebras). We shall also prove that for a large class of commutative WSC Banach algebras with b.a.i., Arens regularity is equivalent to finite dimensionality of the algebra. As an application of the method used to prove the main result, we prove that for any continuous homomorphism $h$ from an Arens regular Banach algebra $A$ with a b.a.i. into a WSC one $B$, the subalgebra $\overline{h(A)}$ is unital. This implies, for example, that, if the algebra $B$ has no idempotent different from zero, then there exists no nontrivial continuous homomorphism from $A$ into $B$.

\section{NotATION AND PRELIMINARY RESUlts}

Our notation and terminology are quite standard and, for Banach algebras, are those of Bonsall and Duncan [B-D]. For a Banach space $X$, we denote by $X^{*}$ its continuous dual, and we always consider $X$ as naturally embedded into its second dual. For $x \in X$ and $f \in X^{*}$, we denote by $\langle x, f\rangle$ the natural duality between $X$ and $X^{*}$.

Let $A$ be a Banach algebra. A bounded net $\left(e_{\alpha}\right)_{\alpha \in I}$ in $A$ is a bounded approximate identity (=b.a.i.) if, for each $a$ in $A, a e_{\alpha} \rightarrow a$ and $e_{\alpha} a \rightarrow a$. For $a$ in $A$ and $f$ in $A^{*}$, we denote by $f . a$ and $a . f$, respectively, the functionals on $A$ defined by $\langle f . a, b\rangle=\langle f, a b\rangle$ and $\langle a . f, b\rangle=\langle f, b a\rangle$. We denote by $A^{*} A$ and $A A^{*}$, respectively, the subspaces

$$
\left\{f . a: a \in A \text { and } f \in A^{*}\right\}, \quad\left\{a . f: a \in A \text { and } f \in A^{*}\right\}
$$

of $A^{*}$. If $A$ has a b.a.i., then, by Cohen's Factorization theorem [H-R, 32.22], the spaces $A^{*} A$ and $A A^{*}$ are closed in $A^{*}$. We need only the following two results about Arens regular Banach algebras:

a) If $A$ is Arens regular and has a b.a.i., then $A^{*} A=A A^{*}=A^{*}$ [U1, Corollary $3.2]$.

b) Closed subalgebras of an Arens regular algebra are Arens regular.

Finally, throughout the paper the term "unital Banach algebra" and "Banach algebra with a unit element" are used interchangeably.

\section{The MAIN RESUlts}

In this section we prove that, for a nonunital Banach algebra $A$, the properties "Arens regularity", "b.a.i." and "WSC" cannot coexist together. If the algebra $A$ is separable, the proof of this result is easy, as the next lemma shows.

Lemma 3.1. Any WSC Arens regular Banach algebra with a sequential b.a.i. is unital.

Proof. Let $A$ be a WSC Arens regular Banach algebra with a sequential b.a.i. $\left(e_{n}\right)_{n \in N}$. Then $A A^{*}=A^{*} A=A^{*}$. Let $f$ be an element in $A^{*}$. Then $f=g$. $a$ for some $g \in A^{*}$ and $a \in A$. So $\left\langle f, e_{n}\right\rangle=\left\langle g, a e_{n}\right\rangle \rightarrow\langle g, a\rangle$, as $n \rightarrow \infty$. This shows that the sequence $\left(e_{n}\right)_{n \in N}$ is weakly Cauchy in $A$. The algebra $A$ being WSC, the sequence $\left(e_{n}\right)_{n \in N}$ converges weakly to an element, say $e$, of $A$. For $f \in A^{*}$ and $a \in A$, we have

$$
\langle f, a e\rangle=\lim \left\langle f . a, e_{n}\right\rangle=\lim \left\langle f, a e_{n}\right\rangle=\langle f, a\rangle,
$$

and so $e a=a$. Similarly, $a e=a$, so that $e$ is the unit element of $A$. 
The next lemma plays an essential role in the proof of the main result. For that reason, although one version of it is proved in [U1], we give here a complete (and different) proof of it.

Lemma 3.2. Let $A$ be any Banach algebra with a b.a.i. Then given any separable subspace $X$ of $A$, there exists a separable subalgebra $B$ of $A$ with a sequential b.a.i. such that $B \supseteq X$.

Proof. Let $\left(x_{n}\right)_{n \geqslant 1}$ be a dense sequence in $X$. Let $\left(e_{\alpha}\right)_{\alpha \in I}$ be a b.a.i. in $A$ with $\left\|e_{\alpha}\right\| \leq C$, for some $C$. We define inductively a sequence $\left(e_{n}\right)_{n \geqslant 1}$ satisfying the inequality $\left\|e_{n}\right\| \leq C$ for all $n \geq 1$, as follows. We choose $e_{1} \in A$ such that

$$
\left\|e_{1} x_{1}-x_{1}\right\|<1, \quad\left\|x_{1} e_{1}-x_{1}\right\|<1 \text { and }\left\|e_{1}\right\| \leq C \text {. }
$$

The elements $e_{1}, e_{2}, \ldots, e_{n}$ being chosen, we choose $e_{n+1}$ such that, for $1 \leq i \leq n$,

$$
\begin{gathered}
\left\|x_{i} e_{n+1}-x_{i}\right\|<1 /(n+1), \quad\left\|e_{n+1} x_{i}-x_{i}\right\|<1 /(n+1), \\
\left\|e_{i} e_{n+1}-e_{i}\right\|<1 /(n+1), \quad\left\|e_{n+1} e_{i}-e_{i}\right\|<1 /(n+1) \text { and }\left\|e_{n+1}\right\| \leq C .
\end{gathered}
$$

Set $Y=\left\{x_{n}: n \geq 1\right\} \cup\left\{e_{n}: n \geq 1\right\}$. For $a \in Y, e_{n} a \rightarrow a$ and $a e_{n} \rightarrow a$, as $n \rightarrow \infty$. If we take for $B$ the closed subalgebra of $A$ generated by $Y$, then $B$ is separable and contains $X$. The sequence $\left(e_{n}\right)_{n \geqslant 1}$ is a b.a.i. for $B$ and is bounded by the same constant $C$ that bounds the net $\left(e_{\alpha}\right)_{\alpha \in I}$.

Now we can prove the main result of the paper.

Theorem 3.3. Let $A$ be a nonunital Banach algebra with a b.a.i. Then $A$ cannot be both WSC and Arens regular.

Proof. Assume on the contrary that $A$ is both WSC and Arens regular. To obtain a contradiction, we shall construct a sequence of linearly independent idempotents $\left(e_{n}\right)_{n \geq 1}$ in $A$ satisfying the relations $e_{m} e_{n}=e_{n} e_{m}=e_{\min \{n, m\}}$, and we shall show that the subalgebra of $A$ generated by them is finite dimensional. To construct such a sequence, we start with an arbitrary separable subspace $X(X \neq\{o\})$ of $A$. By Lemma 3.2, $X$ is contained in a separable subalgebra $A_{1}$ of $A$ with a sequential b.a.i., bounded by the same constant $C$ that bounds the b.a.i. of $A$. The algebra $A_{1}$, being WSC and Arens regular, is unital by Lemma 3.1. Let $e_{1}$ be the unit element of $A_{1}$. Then $\left\|e_{1}\right\| \leq C$ since the b.a.i. of $A_{1}$ converges weakly to $e_{1}$ (see the proof of Lemma 3.1). Let $B_{1}=\left\{a \in A: a e_{1}=e_{1} a=a\right\}$ be the largest subalgebra of $A$ that has $e_{1}$ as the unit element. Since $A$ is not unital, $B_{1} \neq A$. Let $x_{1}$ be an arbitrary element in $A \backslash B_{1}$, and let $X_{1}=\operatorname{Span}\left\{x_{1}, e_{1}\right\}$. For the same reason as above $X_{1}$ is contained in a unital subalgebra $A_{2}$ of $A$. Let $e_{2}$ be the unit element of $A_{2}$. Observe that since $x_{1}$ is not in $B_{1}, e_{2} \notin B_{1}$. Consequently, $e_{1}$ and $e_{2}$ are linearly independent and $e_{1} e_{2}=e_{2} e_{1}=e_{1}$. Continuing this process, we obtain a linearly independent sequence $\left(e_{n}\right)_{1 \geq 1}$ of idempotents in $A$ bounded by the constant $C$ and such that $e_{i} e_{j}=e_{j} e_{i}=e_{\min \{i, j\}}$ for $i, j \geq 1$.

Let $B=\overline{\operatorname{Span}}\left\{e_{n}: n \geq 1\right\}$. Then $B$ is a commutative, separable subalgebra of $A$. For any element $x=\Sigma_{i=1}^{p} \lambda_{i} e_{i}$ in $\operatorname{Span}\left\{e_{n}: n \geq 1\right\}$ and for $n>p, x e_{n}=e_{n} x=x$. It follows that the sequence $\left(e_{n}\right)_{n \geq 1}$ is a b.a.i. for the algebra $B$. The algebra $B$, being WSC and Arens regular, is unital by Lemma 3.1. Let $e$ be the unit element of $B$. Since $\left(e_{n}\right)_{n \geq 1}$ is a b.a.i. for $B$ and $e e_{n}=e_{n}, e_{n} \rightarrow e$ in norm in $B$, as $n \rightarrow \infty$. Hence there exists an integer $N$ such that for $n \geq N, \quad\left\|e_{n}-e\right\|<1$. Since $e_{n}-e$ is an idempotent, the inequality $\left\|e_{n}-e\right\|<1$ is possible only if $e_{n}-e=0$. Hence for 
$n \geq N, \quad e_{n}=e$ and the algebra $B$ is finite dimensional, contracting the fact that the sequence $\left(e_{n}\right)_{n \geq 1}$ is linearly independent. This contradiction proves that for $n \geq N, B_{n}=A$ so that $A$ is unital, which contradicts the hypothesis and finishes the proof.

This theorem applied to the group algebra $L^{1}(G)$ of a locally compact group $G$ falls short of giving Young's theorem [Y] asserting that the algebra $L^{1}(G)$ is Arens regular if and only if the group $G$ is finite. Theorem 3.3. implies only that $G$ is discrete in the case where the algebra $L^{1}(G)$ is Arens regular.

Next we present a less general but more precise result. We recall that any amenable Banach algebra and its cofinite closed ideals (i.e., ideals whose codimensions are finite) have, b.a.i.'s [Cu-L, Corollary 3.8]. This is also the case for certain nonamenable algebras such as the Fourier algebra $A(G)$ of an amenable group $G[\mathrm{~F}]$. For the sake of briefness, we shall say that a Banach algebra $A$ has b.a.i.p.(=bounded approximate identity property) if $A$ and its cofinite closed ideals have b.a.i.'s.

Theorem 3.4. Let $A$ be a WSC commutative Banach algebra with b.a.i.p. Then $A$ is Arens regular if and only if it is semisimple and finite-dimensional.

Proof. It is enough to prove the direct implication. To prove this, suppose that $A$ is Arens regular. Then, by the preceding theorem, $A$ and its cofinite closed ideals are unital. In particular, the Gelfand spectrum $\Sigma$ of $A$ is compact. We want to prove that $\Sigma$ is discrete. To prove this, for $f \in \Sigma$, denote by $e_{f}$ the unit element of the ideal ker $f$. Since $e_{f}$ is an idempotent and, for $g \in \Sigma \backslash\{f\}, f$ and $g$ are linearly independent, $\left\langle e_{f}, g\right\rangle=1$. The function $\widehat{e_{f}}: \Sigma \rightarrow C, \widehat{e_{f}}(g)=$ $\left\langle e_{f}, g\right\rangle$, being continuous and $\left\langle e_{f}, g\right\rangle=1$ for all $g \in \Sigma \backslash\{f\}$, whereas $\left\langle e_{f}, f\right\rangle=0$, we conclude that $f$ is an isolated point of $\Sigma$. This being true for each $f \in \Sigma$, we conclude that $\Sigma$ is discrete, so finite. Let $\Sigma=\left\{f_{1}, f_{2}, \ldots, f_{n}\right\}$ and $J=\operatorname{ker} f_{1} \cap$ $\ldots \cap \operatorname{ker} f_{n}$. Then the ideal $J$ is the radical of the algebra $A$ and the element $u=e_{f_{1}} \cdot e_{f_{2}} \ldots . e_{f_{n}}$ is the unit element of $J$. Since the radical of a Banach algebra cannot contain a nontrivial idempotent element, $u$ must be zero. It follows that $A$ is semisimple. Since $\Sigma$ if finite, we conclude that $A$ is finite-dimensional.

It follows that, for an amenable locally compact group $G$, the Fourier algebra $A(G)$ is Arens regular if and only if $G$ is finite, a result first proved by Lau and Wong $[\mathrm{L}-\mathrm{W}]$.

\section{Some APPLiCATiOns}

In this section we present some applications of the theorems proved in the preceding chapter to the study of homomorphisms between Banach algebras. These theorems can also be used to prove that nonunital, Arens regular Banach algebras with b.a.i.'s cannot be WSC. A typical example of this is the algebra of the compact operators on a reflexive, infinite-dimensional Banach space with the (Grothendieck) approximation property. This kind of results being well known, we include only one of them as a sample.

The next theorem is actually equivalent to Theorem 3.3. But thinking that both theorems and their proofs are of independent interest, we have included both of them. The reader will observe that in this and the following results we do not make any weak compactness assumption on the homomorphisms in question. 
Theorem 4.1. Let $A$ be an Arens regular Banach algebra with a b.a.i., and let $B$ be a WSC Banach algebra. Then, for any (nontrivial) continuous homomorphism $h: A \rightarrow B$, the algebra $\overline{h(A)}$ is unital.

Proof. Let $h: A \rightarrow B$ be a nontrivial continuous homomorphism. We can and do suppose that $\overline{h(A)}=B$. If $B$ is unital, we have nothing to prove. So we suppose that $B$ is not unital and try to obtain a contradiction. At this point we remark that the equality $B=\overline{h(A)}$ does not imply that the algebra $B$ is Arens regular, so Theorem 3.3 does not apply directly. To proceed with the proof, we first remark that the algebra $A$, being Arens regular and having a b.a.i., by Lemma 3.1, has lots of nontrivial separable subalgebras with b.a.i.'s, bounded by the same constant, say $C$, that bounds the b.a.i. of $A$. Let $\left(A_{1},\left(d_{n, 1}\right)\right)$ be one of them. As the algebra $A_{1}$ is Arens regular, $A_{1} A_{1}^{*}=A_{1}^{*}$. It follows that the sequence $\left(d_{n, 1}\right)_{n \in N}$ is weakly Cauchy in $A_{1}$. As $h$ is continuous and $B$ is WSC, the sequence $\left(h\left(d_{n, 1}\right)\right)_{n \in N}$ converges weakly to some element $e_{1}$ of $B$. This element $e_{1}$ is the unit element of the subalgebra $\overline{h\left(A_{1}\right)}$ of $B$, and $\left\|e_{1}\right\| \leq C\|h\|$. Let $B_{1}=\left\{b \in B: b e_{1}=e_{1} b=b\right\}$ be the largest subalgebra of $B$ having $e_{1}$ as the unit element. Since $B$ is not unital, $B_{1} \neq B$. Let $b_{1}$ be an element in $B \backslash B_{1}$. Since $\overline{h(A)}=B, A$ has a separable subalgebra $A_{2}$ with a sequential b.a.i. such that $b_{1} \in \overline{h\left(A_{2}\right)}$. Let $e_{2}$ be the unit element of $\overline{h\left(A_{2}\right)}$. Then $e_{2} \notin \overline{h\left(A_{1}\right)}$ and $e_{1} e_{2}=e_{2} e_{1}=e_{1}$. In this way we construct inductively a sequence of separable subalgebras $\left(A_{k},\left(d_{n, k}\right)\right)$ of $A$ with sequential b.a.i'.s and a sequence of idempotents $\left(e_{n}\right)_{n \geq 1}$ in $B$ such that:

(i) $A_{1} \subset A_{2} \subset \ldots \subset A_{k} \subset \ldots$;

(ii) $e_{n} \in \overline{h\left(A_{n}\right)}$ but $e_{n+1} \notin \overline{h\left(A_{n}\right)}$ for $n \geq 1$;

(iii) $e_{i} e_{j}=e_{j} e_{i}=e_{\min \{i, j\}}$ and, for all $n \geq 1, \quad\left\|e_{n}\right\| \leq C\|h\|$.

Let $D=\overline{\bigcup_{k \geq 1} A_{k}}$. Then $D$ is a separable subalgebra of $A$ and the double sequence $\left(d_{n, k}\right)_{n \geq 1, k \geq 1}$ is a b.a.i. for $D$. It follows that the algebra $E=\overline{h(D)}$ is unital. Let $e$ be its unit element.

On the other hand, since $e_{n}$ is the unit element of $\overline{h\left(A_{n}\right)}$ and $E=\overline{\bigcup_{k \geq 1} h\left(A_{k}\right)}$, the sequence $\left(e_{n}\right)_{n \geq 1}$ is a b.a.i. for the algebra $E$. Since $e e_{n}=e_{n}, e_{n} \rightarrow e$, as $n \rightarrow$ $\infty$. Hence, for $n$ large, $\left\|e-e_{n}\right\|<1$. However, since $e-e_{n}$ is an idempotent, this is possible only if $e_{n}=e$ for $n$ large. But the equalities $e_{n}=e_{n+1}=\ldots=e$ are not possible either since, by (ii), the $e_{n}$ 's are linearly independent. This contradiction proves that the algebra $B=\overline{h(A)}$ is unital, and so completes the proof.

Now we present some applications of the preceding three theorems. For results closely related to the results below we refer the reader to the papers [G-R-W] and [U2].

Corollary 4.2. Let $A$ be an Arens regular Banach algebra with b.a.i.p., and let $B$ be a WSC Banach algebra. If one of the algebras is commutative and $h: A \rightarrow B$ is a continuous homomorphism, then the algebra $\overline{h(A)}$ is semisimple and finitedimensional.

Proof. We can and do suppose that $\overline{h(A)}=B$ and the algebra $B$ is commutative. By the preceding theorem the algebra $B$ is unital. It follows that its Gelfand spectrum $\Sigma_{B}$ is compact. Let $f \in \Sigma_{B}$ be a multiplicative functional on $B$. Then, as one can easily see (see the proof of Lemma 2.2. in [G-R-W]), $h^{-1}(\operatorname{ker} f$ ) is a cofinite closed ideal and $\overline{h\left(h^{-1}(\operatorname{ker} f)\right)}=\operatorname{ker} f$. Since the algebra $A$ has b.a.i.p., the ideal ker $f$ has a b.a.i. Hence, by the preceding theorem, the ideal ker $f$ is unital. 
Now, exactly as in the end of the proof of Theorem 3.4, we show that $\Sigma_{B}$ is finite, $B$ is semisimple and finite-dimensional.

We remark that, since the range of the Fourier transform $\Gamma: L^{1}(R) \rightarrow C_{o}(R)$ is infinite dimensional, it is not possible to reverse the roles of $A$ and $B$ in the preceding corollary. However, we have the following result.

Corollary 4.3. Let $A$ be a commutative Banach algebra with b.a.i.p., and let $B$ be a WSC, Arens regular Banach algebra. Then, for any continuous homomorphism $h: A \rightarrow B$, the algebra $\overline{h(A)}$ is semisimple and finite-dimensional.

Proof. Let $h: A \rightarrow B$ be a continuous homomorphism. We can and do suppose that $\overline{h(A)}=B$. Then $B$ is commutative and, by Theorem 3.3, is unital. As in the proof of the preceding corollary, we show that for each $f \in \Sigma_{B}$, $\operatorname{ker} f$ has a b.a.i. Hence, again by Theorem 3.3, ker $f$ is unital. From this, exactly as in the proof of the preceding corollary, we show that $B$ is semisimple and finite-dimensional.

In the next corollary, which is the final result of the paper, $H$ is a Hilbert space and $L(H)$ is the $C^{*}$ - algebra of the bounded operators on $H$.

Corollary 4.4. Let $A$ be a nonunital closed subalgebra of $L(H)$ with a b.a.i. Then $A$ is not WSC.

Proof. The algebra $A$ is Arens regular, being a closed subalgebra of the Arens regular algebra $L(H)$. By Theorem 3.3, $A$ is not WSC.

At this point we recall that the algebras $\ell^{p}(1 \leq p \leq \infty)$ are function algebras. (i.e., they are isomorphic to closed subalgebras of $L(H)$ ).

\section{ACKNOWLEDGMENT}

The author is grateful to the referee for his generous suggestions concerning the presentation of the paper.

\section{REFERENCES}

[B-D] F.F. Bonsall and J. Duncan, Complete normed algebras, Springer-Verlag (1973), NewYork. MR 54:11013

[C-Y] I.G. Craw and N. Young, Regularity of multiplication in weighted group and semigroup algebras, Quart. J. Math. Oxford (2) 25 (1974), 351-381. MR 51:1282

[Cu-L] P. C. Curtis Jr. and R.J. Loy, The structure of amenable Banach algebras, J. London Math. Soc. (2) 40 (1989), 89-104. MR 90k:46114

[D] C.F. Dunkl, The measure algebra of a locally compact hypergroup, Trans. Amer. Math. Soc. 179 (1973), 331-348. MR 47:9171

[E] P. Eymard, L'Algebre de Fourier d'un groupe localement compact, Bull. Soc. Math. France 92 (1964), 181-236. MR 37:4208

[F] B. Forrest, Amenability and the structure of the algebra $A_{p}(G)$, Trans. Amer. Math. Soc. 343 (1994), 233-243. MR 94g:43001

[G-R-W] J.E. Galé, T.J Ransford and M.C. White, Weakly compact homomorphisms, Trans. Amer. Math. Soc. 331 (1992), 815-824. MR 92h:46074

[H-R] E. Hewitt and K. Ross, Abstract Harmonic Analysis. Vol II, Springer-Verlag (1970), New-York. MR 41:7378

[J] R.I. Jewett, Spaces with an abstract convolution of measures, Advances in Math. 18 (1975), New-York. MR 52:14840

[L-W] A.T. Lau and J.C.S. Wong, Weakly almost periodic elements in $L_{\infty}(G)$ of a locally compact group, Proc. Amer. Math. Soc. 107 (1989), 1031-1036. MR 90m:43006

[U1] A. Ülger, Arens regularity sometimes implies the RNP, Pacific J. Math. 143 (1990), 377-399. MR 91f:46067 
[U2] A. Ülger, Some results about the spectrum of commutative Banach algebras under the weak topology and applications, Monaths. Für Math. 121 (1996), 353-379. MR 98a:46058

[Y] N. Young, The irregularity of multiplication in group algebras, Quart. J. Math. Oxford (2) 24 (1973), 59-62. MR 47:9290

Department of Mathematics, Koç University, 80860 Istinye-Istanbul, Turkey

E-mail address: aulger@ku.edu.tr 